\title{
Announcing cIMPACT-NOW: the Consortium to Inform Molecular and Practical Approaches to CNS Tumor Taxonomy
}

\author{
David N. Louis ${ }^{1} \cdot$ Ken Aldape $^{2} \cdot$ Daniel J. Brat ${ }^{3}$ David Capper ${ }^{4,5} \cdot$ David W. Ellison $^{6} \cdot$ Cynthia Hawkins $^{7} \cdot$ \\ Werner Paulus $^{8} \cdot$ Arie Perry $^{9}$ - Guido Reifenberger ${ }^{10,11} \cdot$ Dominique Figarella-Branger $^{12} \cdot$ Pieter Wesseling $^{13,14}$. \\ Tracy T. Batchelor ${ }^{15} \cdot$ J. Gregory Cairncross ${ }^{16} \cdot$ Stefan M. Pfister $^{17,18,19} \cdot$ Stefan Rutkowski $^{20} \cdot$ Michael Weller $^{21}$. \\ Wolfgang Wick $^{18,22,23} \cdot$ Andreas von Deimling ${ }^{4,5}$
}

Published online: 1 December 2016

(C) Springer-Verlag Berlin Heidelberg 2016

The recent publication of the 2016 World Health Organization Classification of Tumors of the Central Nervous System (2016 CNS WHO) represents a significant advance in the classification of human brain tumors [1]. For the first time, a CNS WHO classification defines diagnostic entities by combining molecular and histological information. In doing so, the classification facilitates more precise diagnosis of well-understood entities and clearer designation of less-understood entities, which will in turn allow further study and likely future advances in their classifications.

T. T. Batchelor, J. G. Cairncross, S. M. Pfister, S. Rutkowski, M. Weller, and W. Wick: cIMPACT-NOW Clinical Advisory Panel.

David N. Louis

dlouis@mgh.harvard.edu

1 Pathology Service, Department of Pathology, Massachusetts General Hospital, Harvard Medical School, WRN225, 55 Fruit Street, Boston, MA 02114, USA

2 Department of Pathology, Princess Margaret Hospital, Toronto, Canada

3 Department of Pathology and Laboratory Medicine, Emory University School of Medicine, Atlanta, GA, USA

4 Department of Neuropathology, Institute of Pathology, Ruprecht-Karls-University, Heidelberg, Germany

5 Clinical Cooperation Unit Neuropathology, German Cancer Research Center (DKFZ) and German Cancer Consortium (DKTK), Heidelberg, Germany

6 Department of Pathology, St. Jude Children's Research Hospital, Memphis, TN, USA

7 Department of Paediatric Laboratory Medicine, The Hospital for Sick Children, University of Toronto, Toronto, Canada

8 Institute of Neuropathology, University Hospital Munster, Münster, Germany
Such updates have broad implications in a variety of settings: the care of individual patients; the conduct and interpretation of clinical trials; the analysis of basic scientific experiments; the elucidation of population-based disease trends that may implicate specific etiologies; and the allocation of resources by governments and health insurers to support health care [2]. As a result, the 2016 CNS WHO attempted to be responsive to the needs of a wide variety of constituencies that depend on formal classifications for their work: clinicians in various neuro-oncological disciplines, scientists involved in translational and basic research on brain tumors, epidemiologists tracking disease patterns and searching for etiological clues, and those

9 Department of Pathology, University of California San Francisco, San Francisco, CA, USA

10 Department of Neuropathology, Heinrich Heine University, Düsseldorf, Germany

11 German Cancer Consortium (DKTK) Partner Site Essen/ Duesseldorf, Düsseldorf, Germany

12 Department of Pathology and Neuropathology, La Timone Hospital, Aix Marseille University, Marseille, France

13 Department of Pathology, VU University Medical Center, Amsterdam, The Netherlands

14 Department of Pathology, Princess Máxima Center for Pediatric Oncology and University Medical Center Utrecht, Utrecht, The Netherlands

15 Department of Neurology, Massachusetts General Hospital, Harvard Medical School, Boston, MA, USA

16 Department of Clinical Neurosciences, University of Calgary, Calgary, Canada

17 Division of Pediatric Neurooncology, German Cancer Research Center (DKFZ) Heidelberg, Heidelberg, Germany 
public and private agencies who fund healthcare for populations that have nervous system tumor patients.

To balance these needs, WHO classifications are updated periodically, with the intervals between updates varying as a result of many factors, including the allocation of funds to support each new edition series of classifications. However, the different constituencies may have distinct preferences and needs for the frequency and timing of updates. For example, for those in research, demands for revisions are common and rapid updates appreciated; for those tracking disease trends, on the other hand, continuity of classification systems is essential, and too frequent updates are disadvantageous; and for those managing patients, the desire may be a happy medium between updating too often versus not often enough. As in the Goldilocks story, one needs to try multiple options to find a right fit—but the final fit may not be perfect for all.

Finding the right fit for how often CNS WHO classifications should be updated has been made more difficult over the past two decades, since there has clearly been an acceleration in our understanding of the molecular characteristics of human nervous system tumors and in our appreciation for the clinicopathological correlates of these molecular changes. Indeed, this accelerated understanding drove the decision for an earlier, 4th edition update of the CNS WHO rather than waiting longer for a 5th edition [3]. However, there is still concern that the pace of change in the field creates a need to evaluate classification progress faster than is possible through standard WHO updates.

In response to this concern, we announce an initiative to evaluate and recommend proposed changes to future CNS tumor classifications: cIMPACT-NOW, the Consortium to Inform Molecular and Practical Approaches to CNS Tumor Taxonomy. The goal of cIMPACT-NOW is to facilitate input and consensus review of novel diagnostically relevant data and determine how such information can be practically incorporated into future CNS tumor classifications.

18 German Cancer Consortium (DKTK) Core Center Heidelberg, Heidelberg, Germany

19 Department of Pediatric Oncology, Hematology and Immunology, Heidelberg University Hospital, Heidelberg, Germany

20 Department of Pediatric Haematology and Oncology, University Medical Center Hamburg-Eppendorf, Hamburg, Germany

21 Department of Neurology, University Hospital Zürich, Zurich, Switzerland

22 Neurology Clinic and National Center for Tumor Diseases (NCT), University Hospital Heidelberg, Heidelberg, Germany

23 Clinical Cooperation Unit Neurooncology, German Cancer Research Center (DKFZ), Heidelberg, Germany
While it is understood that the major impact on international brain tumor classification comes about through the WHO classification update process, it is anticipated that the cIMPACT-NOW initiative will "see impact" in selected tumor types and in time periods between the WHO classification updates. Thus, the cIMPACT-NOW updates are not intended to supplant the existing WHO classification, nor are they in any way part of the official WHO process; rather, the cIMPACT-NOW updates are intended to provide possible guidelines for practicing diagnosticians as well as possible guideposts for future WHO classification updates.

cIMPACT-NOW initially involves neuropathologists who played leading roles in the 2016 CNS WHO classification as well as a representative Clinical Advisory Panel, which will also suggest ad hoc clinical consultants for each major review topic and will review recommendations. In addition, the group plans to cycle its membership, primarily by bringing along new junior members. It is anticipated that cIMPACT-NOW will solicit, circulate, and evaluate suggestions for topics in at least yearly intervals and that consensus recommendations on these topics will also be issued about once a year. Generation of these proposals will be accomplished through a process that involves dynamic working groups, each tackling a specific topic judged to be ready for evaluation. Significantly, cIMPACT-NOW is sponsored by the International Society of Neuropathology (ISN), reflecting the central role played by neuropathologists in CNS tumor classification and the substantial effect that new classifications play in the daily activities of neuropathologists. It is expected that the group will promptly publish its recommendations; after appropriate review, each consensus publication will be in Acta Neuropathologica with an accompanying commentary focusing on the neuropathological implementation of the recommendations, in Brain Pathology, the official ISN journal.

cIMPACT-NOW, prompted by the quickening pace of change in the field, represents a change in the way the international neuro-oncology community approaches tumor classification. Like the official WHO classification process, however, it is hoped that cIMPACT-NOW will provide consensus recommendations that will improve clinical, experimental, and epidemiological approaches to human nervous system tumors and eventually lead to a cure for these diseases.

\section{References}

1. Louis DN, Ohgaki H, Wiestler OD, Cavenee WK (2016) World Health Organization histological classification of tumours of the central nervous system. International Agency for Research on Cancer, Lyon

2. Louis DN, Perry A, Burger P, Ellison DW, Reifenberger G, von Deimling A, Aldape K, Brat D, Collins VP, Eberhart C et al 
(2014) International Society Of Neuropathology-Haarlem consensus guidelines for nervous system tumor classification and grading. Brain Pathol 24:429-435. doi:10.1111/bpa.12171
3. Louis DN, Perry A, Reifenberger G, von Deimling A, FigarellaBranger D, Cavenee WK, Ohgaki H, Wiestler OD, Kleihues P, Ellison DW (2016) The 2016 World Health Organization Classification of tumors of the central nervous system: a summary. Acta Neuropathol 131:803-820. doi:10.1007/s00401-016-1545-1 